\title{
Dysfunctional doctors - will revalidation help?
}

\author{
John Scarpello
}

\begin{abstract}
Numbers of National Clinical Assessment Service referrals concerning practitioners' performance are much higher for the over-50s, especially in GPs. In $20 \%$ of cases there are concerns with diagnosis that are unlikely to be recognised by revalidation. Accurate and timely diagnosis is fundamental to patient safety, and the results of studies in which GPs were tested using mystery patients are not encouraging. We need to assess pratictioners' competencies in diagnosis throughout their careers. Plans to extend working life may require changes to the clinical responsibilities of older doctors.
\end{abstract}

KEY WORDS: revalidation, diagnostic skills, NCAS, clinical competency, older doctors

\section{Introduction}

The National Clinical Assessment Service (NCAS) was established in 2001 and works with health organisations and individual practitioners when there is a concern about the performance of a doctor. Three-quarters of all providers refer at least one practitioner each year. In 2009 NCAS published an analysis of the first eight years of their work and described the more common features of those doctors referred to them. ${ }^{1}$

They have around 450 cases open at any one time and one-third of these involve practitioners suspended or excluded from NHS work while under investigation. There was a greater geographical variation in referrals from primary care than from the hospital and community $(\mathrm{H}+\mathrm{C})$ organisations and in addition suspension was more variable among general practitioners (GPs). For both $\mathrm{H}+\mathrm{C}$ and primary care the chance of referral is lower among the younger age groups. Referral in employees of $\mathrm{H}+\mathrm{C}$ organisations is five times higher among those aged over 50 years compared with those under 40 years. For GPs over the age of 60 , the referral rate is seven times that of those under 40 years. Overall, around twothirds of the cases involved clinical difficulties, governance or safety issues while $16 \%$ of cases involved misconduct alone. Concerns about diagnostic skills were reported in $20 \%$ of cases and communication with colleagues was the most common behavioural difficulty. Communication with management or with patients/carers was mentioned in $12 \%$. Within hospital practice, general medicine accounted for approximately $7 \%$ and surgery $15 \%$. Perhaps surprisingly, given the high level of litigation claims, obstetrics and gynaecology contributed only $5 \%$.

Following investigation, more than half of excluded $\mathrm{H}+\mathrm{C}$ practitioners eventually resumed work in the same organisation,

John Scarpello, emeritus consultant physician, University Hospital of North Staffordshire although they were often subject to restrictions. By contrast, GPs fared worse, with only about one-third returning to work and more than two-fifths being removed from the performers list. These numbers do not include doctors referred to the General Medical Council (GMC), some of whom will also be suspended or, in the worst cases, erased from the medical register.

\section{Doctors causing concern}

The information from NCAS is important and, while it should be emphasised that as a proportion of the total employed the numbers are small, it is likely to considerably underestimate the number of doctors that should give cause us for concern. At present nearly all referrals to NCAS only take place after lengthy investigation and attempts at resolution by local employers. Moreover, it can take considerable time before long-term issues of clinical competency, communication or team functionality are reported internally and it is probable that a majority are neither recognised nor investigated. Even when concerns about such relevant safety issues as team functionality are investigated, too often the enquiries are confined within a directorate team and may not be drawn to the attention of more senior managers. This is a failure of governance.

\section{Whistle blowing and Invited Service Reviews}

There remains a culture of resistance to 'whistle blowing'. Criticism about the behaviour of doctors is especially difficult for other members of clinical teams, such as nurses. There is a general reluctance to criticise senior staff, especially by their junior colleagues. The NCAS data demonstrates for the first time that there are more likely to be concerns about older clinicians than younger ones. The real numbers may be even higher, since some employers are reluctant to investigate.

Many of these same issues were the reasons given for requesting an Invited Service Review (ISR) by the Royal College of Physicians (RCP), which have been described previously. ${ }^{2}$ The RCP found that requests often occurred late in the process and long after concerns were first raised with management. Frequently trusts had not followed their own policies and processes, especially in cases where clinical competency was concerned, when there was often a reluctance to make a judgement. Long-standing issues around working practices and poor team working were largely ignored and the importance of human factors in delivering safe and effective care was not understood. Dysfunctional doctors, whether in primary or secondary care, can cause considerable harm before being recognised and need to be identified earlier. 


\section{How do you know if your doctor is a good one?}

All doctors make mistakes and, while only some of these will result in serious harm, some patients will be unlucky. Care will vary by provider and therefore it is important that outcome data is collected and analysed in order that consistent outliers are detected and corrective measures undertaken. In England hospital mortality data is now compared and variances identified. In contrast there is little comparable data from primary care, despite the very much greater number of patient contacts.

How can we help to ensure that all doctors maintain at least average standards in their diagnostic skills, investigation and treatment, including safe prescribing? This question has received added impetus in recent years following several high profile incidences of poor practice in which care was compromised. One of the first concerned the failings of child heart surgery at Bristol Royal Infirmary in the late 1990s. A series of major reviews and official reports followed, perhaps the most influential of which was the Shipman Inquiry, which led to calls for annual appraisal of all doctors, and, now, to revalidation. ${ }^{3}$

In 2006 the publication Good doctors safer patients ${ }^{4}$ raised the issue of how we could identify substandard practice, especially among GPs, many of whom are self employed as independent practitioners, and where it is more difficult to review information about the quality and outcomes of the care they provide than for employed doctors in secondary care. This is despite the better electronic patient records used by GPs compared with those found in most centres of secondary care provision. The problems of monitoring the quality of healthcare by GPs was highlighted by a controversial Channel 4 Dispatches broadcast in 2010..$^{5}$ They used actors as undercover 'mystery' patients primed with common symptoms that ought to have suggested the diagnosis of potentially serious conditions ('red flags') and which required immediate investigation or referral. The histories of these 'mystery' patients included pointers to possible intestinal cancer, which often presents late to specialist care. The results of this non-scientific sample were not encouraging and demonstrated inadequate clinical diagnostic skills in a number of cases. They were discussed by Aneez Esmail, a former medical advisor to the Shipman enquiry, who believes that there is no system presently available to confirm the safety and clinical skills of GPs. ${ }^{6,7}$ More extensive investigation by the Kings Fund also found disturbing variations across a range of diagnostic indicators and treatment options among GPs, including failure to recognise common presentations of serious illness. ${ }^{8}$ It seems likely that similar shortcomings would be found for some consultants.

Although doctors employed in the $\mathrm{H}+\mathrm{C}$ sectors work in less isolation than some GPs, the effective demise of the traditional 'firm' structure in hospital practice may add to the risk of poor clinical practice or inappropriate communication skills remaining unnoticed or unreported, since more consultants now work single-handed in clinics and on wards. There is much to commend a system that encourages shared patient care by consultants with joint decision-making and audit of outcomes, in particular shared mortality and morbidity meetings. These are not yet routine for all specialties and providers.

It is not only a matter of analysing clinicians' skills. As the events at Stafford hospital have highlighted, poor practice may result from poor management. Sub-standard practice has to be challenged wherever it is found. The GMC now expects evidence of poor practice to be brought to the attention of appropriate authorities as part of the duty of care. ${ }^{9}$

\section{Will revalidation make a difference?}

In particular, will revalidation improve diagnostic skills and communication with patients and between clinicians (the reasons given for most referrals to NCAS)? Revalidation, which begins this year and which will be based upon five-year data derived from annual appraisals, has the potential to be one of the most important elements in ensuring safer practice. Appraisal provides the opportunity for clinicians to identify weaknesses, agree plans to update their skills and to show evidence of what they have learnt from patient feedback and how they have dealt with any complaints. The process is designed to strengthen the confidence of the public in the quality and safety of their care. In time it will change the way we are regulated and should lead to improvement in standards of practice.

Since revalidation will be dependent upon the existing and evolving system of annual appraisal, it is reasonable to ask if this will be sufficient to satisfy public demands. Currently, assessment is largely formative and is designed to be supportive. It is reviewed by assessors in different ways and with varying expertise. It presents particular challenges for smaller specialties and where a clinician works for several organisations, perhaps including primary care or single-handed working. The development of revalidation and the role of the RCP in the process was described in Clinical Medicine in $2010^{10}$ and updated in the October 2011 edition of the RCP's magazine Commentary. ${ }^{11}$ Appraisals will need to become more structured and supported by improved information systems for all specialties, since at present clinically relevant information systems are only available in a minority, for example cardiology. There are several online tools available that have been developed by the RCP to help with revalidation, including a useful personal clinical audit tool. ${ }^{11}$

More accurate information will be essential if revalidation is to prove effective in raising standards of practice. It will need to include verifiable data of clinical incident reports, including all complaints received, and show the actions taken to reduce recurrence. This requires the commitment and support of all employing organisations; at present many hospital based information systems will struggle to provide the data. We also need agreement on evidence-based outcome measures, which will vary between medical specialties. A guide to the information required by physicians has been published by the RCP. ${ }^{12}$ It may prove more difficult to collect from GPs given their independent contractual status.

The GMC also requires that appraisers are provided with reliable information from anonymised patient feedback about individual doctors. Validated questionnaires that follow GMC principles 
are available (see for example www.360clinical.com). Analysis of multi-source feedback using questionnaires completed by both patients and doctors' colleagues has been undertaken by the RCP. ${ }^{13}$

Although revalidation based upon annual appraisal is to be welcomed, it is unlikely to provide much evidence concerning diagnostic skills or unsafe practices, unless coincidentally they are mentioned by patients or staff participating in the questionnaires or have been the subject of a complaint or other investigation. This is a serious omission since mistakes in diagnosis are one of the most important patient safety issues and are of great concern to patients. ${ }^{14}$ Outcome data may also fail to identify delayed or poor diagnosis by an individual doctor or service while showing shortcomings across the whole system. For example, the debate over survival figures for cancer in the UK, in which delayed referral may be an important factor in affecting national data, contrasts less favourably with data from other European healthcare systems. Without re-examination (which is considered impracticable and would most likely be fiercely resisted), using questionnaires to test diagnostic skills or even the use of 'mystery' patients, it is difficult to see how we might obtain useful data about this fundamental aspect of patient care. It is curious that even during recruitment for a consultant or GP position there is no assessment of clinical or communication skills, which are assumed to have been confirmed by the relevant certificate of postgraduate training. Indeed, the interview process now usually excludes sight of references, and thus it is difficult to form an opinion about candidates' team-working skills, despite these being vital to all doctors.

\section{Revalidation and dysfunctional behaviour}

Revalidation, at least in its present form, is unlikely to help with dysfunctional behaviour that goes beyond concerns with communication skills with colleagues or patients. As reported by NCAS, this occurs more often among older doctors. Such behaviour, which can be destructive to clinical relationships (especially with more junior colleagues and with other members of the team, including nurses and non-clinical managers), may reflect a general dissatisfaction and a feeling of being ignored. 'What do they know; why should I change? I am the clinician and know what is best for my patients.' Difficult! Will revalidation reduce these issues? More likely they will remain for local employers to address. Clinical teams must help to manage these problems, which are common and adversely affect patient care in many ways.

\section{How else should we help?}

All clinical staff and managers should be encouraged to take responsibility for improving working practices. Concerns with the standards of practice or with inappropriate behaviour must be promptly reported and action taken, since the longer they are left the more difficult they become to correct. Any such reports or investigations should be included in the personal portfolio and discussed at appraisal. Appraisals should seek evidence to demonstrate the effectiveness of the appraisee in team working and should address any difficulties. Human factor training should be incorporated into continuous professional development as it is in many other high-risk industries.

It is clear from NCAS that the challenges associated with meaningful appraisal in primary care are considerably greater, since many practitioners work in small teams and often as independent contractors. The relatively high number of GPs referred to NCAS, especially older doctors, is cause for concern. For all doctors, the abolition of a fixed retirement age presents further challenges to ensure that practice remains safe. Job plans should reflect the changes to competencies at that time. Assessment of diagnostic skills and more evidence of up-to-date practice than is likely to result from the current process will be needed to satisfy the public.

In the end revalidation will only be judged to be successful if it satisfies us that when we visit a doctor we will be seen by someone who is up-to-date in their knowledge and clinical practices and has been able to demonstrate to their peers that they deliver care of the highest standard. As is often said, when we fly we expect to arrive safely and assume that the flight team will all have undergone regular updates and retesting of their technical skills. Many medical procedures are also highly technical and depend upon using complex equipment that is frequently changed by new upgrades. We need assurance that all those using the equipment have the skills to do so. It will be interesting to see how long revalidation based on a formative process of annual appraisal will be considered acceptable.

\section{References}

1 The National Clinical Assessment Service. NCAS Casework. The first eight years' summary. London: NCAS, 2009.

www.ncas.npsa.nhs.uk/resources/publications/caseworkanalyses/

2 Scarpello J. Invited Service Reviews. Clin Med 2010;4:376-8.

3 Reports of The Shipman Inquiry, 2002-2005. www.shipmaninquiry.org.uk/reports.asp [Accessed January 2011].

4 Department of Health. Good doctors, safer patients: Proposals to strengthen the system to assure and improve the performance of doctors and to protect the safety of patients. London: DH, 2006.

5 Can you trust your doctor? www.channel4.com/programmes/ dispatches/4od\#3236726.

6 Esmail A. Secret filming shows deep problems in general practice. BMJ 2011;343:d6392.

7 King's Fund. Improving the quality of care in general practice. London: King's Fund, 2011.

8 Dyer C. Heeding red flags in primary care. BMJ 2011;343:d7315.

9 General medical Council. The state of medical education and practice in the UK, 2011. London: GMC, 2011. www.gmc-uk.org/publications/10471.asp. [Accessed January 2011].

10 Starke I, Brownbridge H. Revalidation: an RCP perspective. Clin Med 2010;4:321-2.

11 Starke I. Q\&A. Commentary October 2011:10-13.

12 Royal College of Physicians. Draft guidance for physicians on supporting information for revalidation, 2011. London: RCP, 2011.

13 General Medical Council. Revalidation: a statement of intent. London: GMC, 2010. www.gmc-uk.org/Revalidation_A_Statement_of_Intent_ Final_version__web_version_pdf_35982397.pdf.

14 Scarpello J. Diagnostic error: the Achilles heel of patient safety? Clin Med 2011;310-11.

Address for correspondence: Dr John Scarpello, 222 Seabridge Lane, Newcastle, Staffs ST5 3LS. Email: john.scarpello@virginmedia.com 